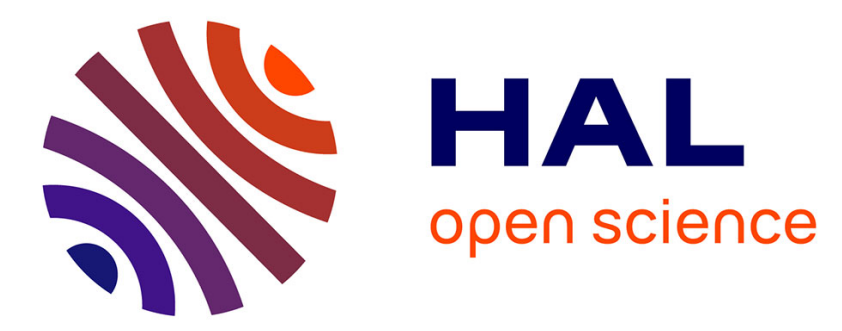

\title{
RUPTURE DYNAMIQUE DE STRATIFIÉS FIBRE DE VERRE
}

\author{
M. Ayax, M. Roux, M. Moureaud
}

\section{To cite this version:}

M. Ayax, M. Roux, M. Moureaud. RUPTURE DYNAMIQUE DE STRATIFIÉS FIBRE DE VERRE. Journal de Physique IV Proceedings, 1991, 01 (C3), pp.C3-687-C3-692. 10.1051/jp4:1991396 . jpa00250542

\section{HAL Id: jpa-00250542 https://hal.science/jpa-00250542}

Submitted on 1 Jan 1991

HAL is a multi-disciplinary open access archive for the deposit and dissemination of scientific research documents, whether they are published or not. The documents may come from teaching and research institutions in France or abroad, or from public or private research centers.
L'archive ouverte pluridisciplinaire HAL, est destinée au dépôt et à la diffusion de documents scientifiques de niveau recherche, publiés ou non, émanant des établissements d'enseignement et de recherche français ou étrangers, des laboratoires publics ou privés. 


\title{
RUPTURE DYNAMIQUE DE STRATIFIÉS FIBRE DE VERRE
}

\author{
M.E. AYAX, M.R. ROUX et M.J. MOUREAUD \\ Creusot-Loire Industrie, Division Creusot-Marrel, Centre de \\ Recherche des Matériaux du Creusot, BP. 56, F-71202 Le Creusot \\ Cedex, France
}

\begin{abstract}
Afin de comparer différents stratifiés à base de fibre de verre en régime dynamique, nous avons utilisé un canon à gaz léger permettant d'envoyer des cylindres à des vitesses allant jusqu'à $500 \mathrm{~m} / \mathrm{s}$.

L'observation microscopique des éprouvettes après essai permet d'identifier les différents mécanismes d'absorption d'énergie (cisaillement, traction, flexion et délaminage).

L'influence du pourcentage de fibre et du grammage a été étudiée à partir des mesures de pénétration et d'aire délaminée.
\end{abstract}

AbstractIn order to compare different woven fiber glass composites we have conducted dynamic impact tests with a light gaz gun. Microscopic investigations allow us to determine the different absorption energy mechanisms (shearing, tension, flexion and delamination). Influence of percentage of fiber and roving weight are discussed in terms of penetration and area of delamination.

\section{1 - INTRODUCTION}

Les matériaux composites et en particulier les stratifiés à base de tissus fibre de verre sont utilisés aujourd'hui dans des domaines où ils peuvent être soumis à des sollicitations dynamiques : chocs, impacts balistiques ...

Afin de déterminer les performances en régime dynamique de différents stratifiés, nous avons utilisé un lanceur à gaz permettant d'envoyer sur une plaque stratifiée un projectile cylindrique de quelques grammes à des vitesses allant jusqu'à $500 \mathrm{~m} / \mathrm{s}$.

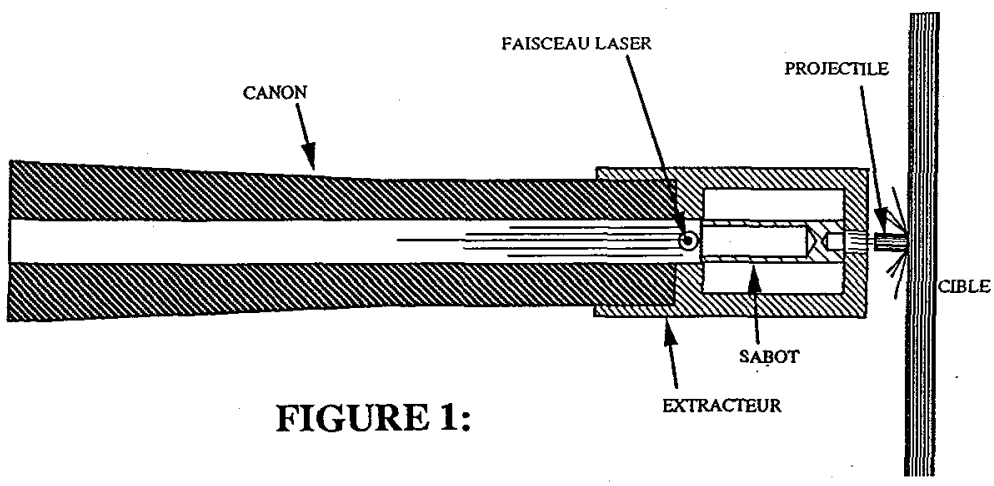




\section{2 - METHODOLOGIE DE L'ESSAI}

Le projectile cylindrique en acier STUB $(\emptyset=6 \mathrm{~mm} ; \mathrm{m}=2,5 \mathrm{~g})$ est guidé à l'intérieur du canon par un sabot en plastique arrêté par un extracteur avant impact.

La mesure de vitesse se fait par rupture d'un faisceau laser juste avant extraction (voir figure 1).

La plaque stratifiée impactée est encastrée dans un cadre rigide ; elle mesure $200 \times 200 \mathrm{~mm}^{2}$; épaisseur variable entre 8 et $12 \mathrm{~mm}$. Elle est constituée de tissus fibre de verre $\mathrm{E}$ dans une matrice polyester.

Les informations recueillies sont : (voir figure 2)

- la vitesse d'impact : V

- la profondeur totale pénétrée : pt

- la profondeur cisaillée : pc

- l'épaisseur non perforée: $n p$

- la surface délaminée en face arrière $: S=\pi D^{2} / 4$.

FIGURE 2:

VUE SUR COUPE AU DROIT DE L'IMPACT

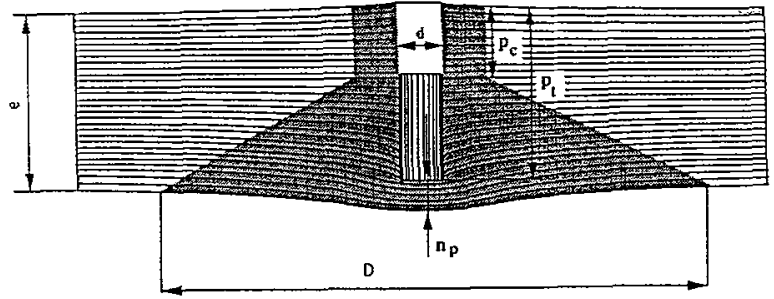

\section{3 - DESCRIPTION DES MECANISMES DE PENETRATION}

Pour ces premiers essais la vitesse d'impact a été choisie de façon à éviter la perforation totale de la plaque testée. L'énergie cinétique incidence $\left(1 / 2 \mathrm{~m} \mathrm{~V}^{2}\right)$ est donc entièrement absorbée au cours du choc.

Les différents mécanismes qui participent à cette absorption d'énergie peuvent être décrits de la façon suivante : (voir figure 3 )

1 - cisaillement des fibres à la périphérie du projectile

2 - rupture par tension des fibres en avant du projectile

3 - délaminage du stratifié en forme de cône

4 - flexion de la plaque lors de l'impact.

Pour les applications recherchées, il est nécessaire d'optimiser les différents paramètres constituant ur stratifié afin d'absorber un maximum d'énergie. 
FIGURE 3:

LOCALISATION DES MECANISMES D'ENDOMAGEMENT

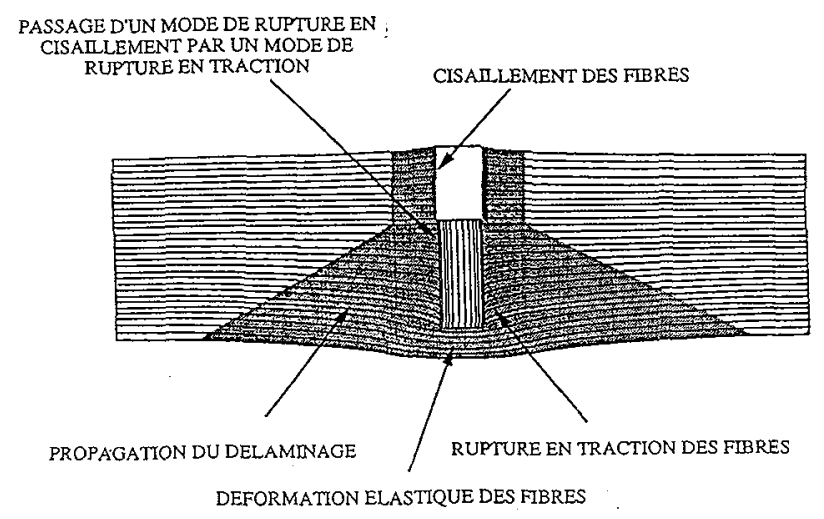

\section{4 - MECANISMES D'ABSORPTION D'ENERGIE}

\section{1 - Cisaillement des fibres}

Le travail nécessaire pour pénétrer dp s'écrit :

$$
\mathrm{W}=\pi \mathrm{d} \cdot \mathrm{Y}_{\mathrm{T}} \cdot \mathrm{p} \mathrm{dp}
$$

L'énergie absorbée pour passer d'une profondeur cisaillée $\mathrm{p}_{1}$ à une profondeur cisaillée $\mathrm{p}_{2}$ s'écrit donc :

$$
E_{C}=\int_{+1}^{+2} \pi d Y_{T} p d p=\frac{1}{2} \pi d Y_{T}\left[p_{2}^{2}-p_{1}^{2}\right]
$$

$\mathrm{d}$ : diamètre du projectile

$\mathrm{Y}_{\mathrm{T}}$ : contrainte de rupture en cisaillement transverse.

\section{2 - Rupture des fibres}

L'énergie nécessaire à la rupture d'une fibre en traction est donnée par la relation :

$$
E_{\mathrm{t}}=1 / 2 \mathrm{~L} \sigma_{\mathrm{r}} \varepsilon_{\mathrm{r}} \simeq 1 / 2 \mathrm{~L} \sigma_{\mathrm{r}}^{2} / \mathrm{E}
$$
$\sigma_{\mathrm{r}}:$ contrainte à rupture
$\varepsilon_{\mathrm{T}}$ : allongement à rupture
$\mathrm{E}$ : module d'élasticité
L : longueur de fibre sollicitée. 


\section{3 - Délaminage du stratifié}

En première approche l'énergie absorbée par délaminage est proportionnelle au volume délaminé dans la structure.

Le mécanisme de délaminage débute lorsque les fibres sont rompues en traction (voir figure 4).

$$
\begin{aligned}
& \text { FIGURE 4: STRATIFIE FIBRE DE VERRE E } 0,5 \mathrm{~kg} / \mathrm{m}^{2} \\
& \text { resine PU } 78 \% \text { fibres } \\
& \text { VITESSE D'IMPACT }=450 \mathrm{~m} / \mathrm{s}
\end{aligned}
$$

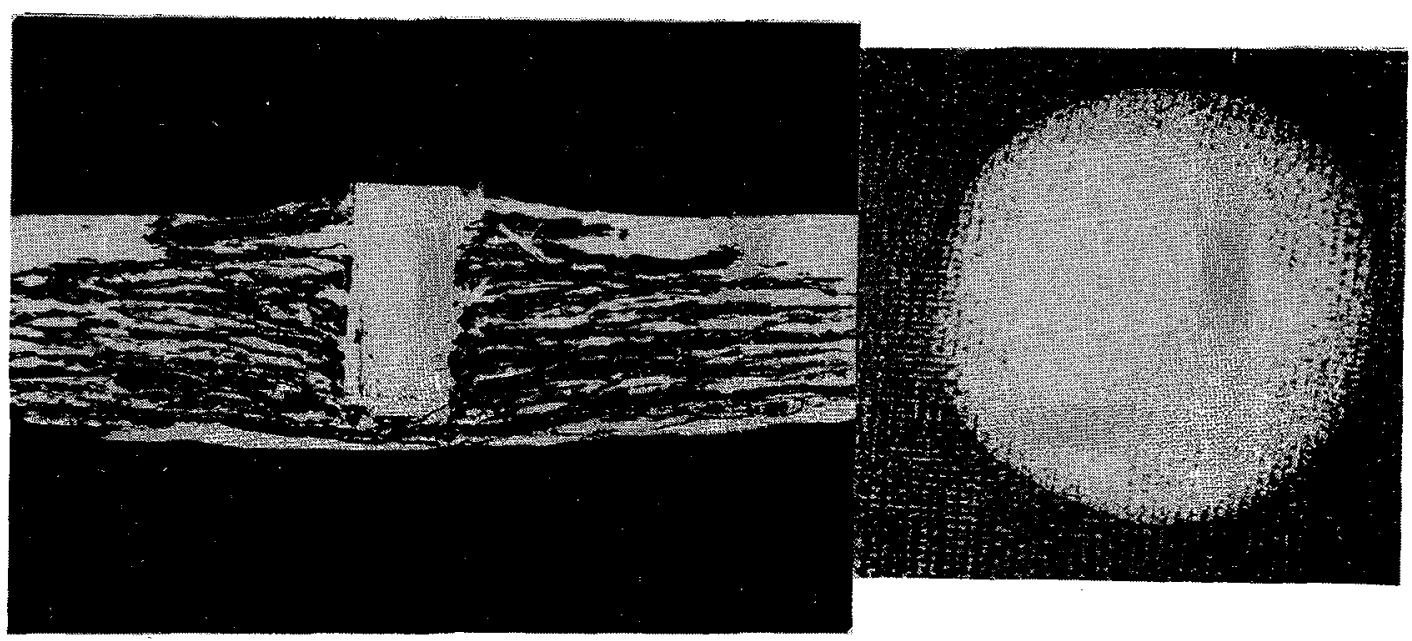

VUE EN COUPE

VUE ARRIERE SURFACE DELAMINEE

L'aire délaminée apparaît en noir sur la photo en coupe et la surface en blanc sur la face arrière.

L'énergie absorbée est donc proportionnelle à : Ed $\alpha \mathrm{S}$. (e - pc).

\section{4 - Flexion de la plaque}

La détermination de l'énergie absorbée par flexion nécessite de connaître les déformations subies au cours de l'impact (caméra ultra-rapide).

\section{5 - RESULTATS EXPERIMENTAUX}

\section{1 - Influence du pourcentage de fibre}

La figure 5 montre que la profondeur cisaillée diminue avec l'augmentation du taux de fibre.

L'absorption d'énergie est donc contrôlée prioritairement par la rupture des fibres. 


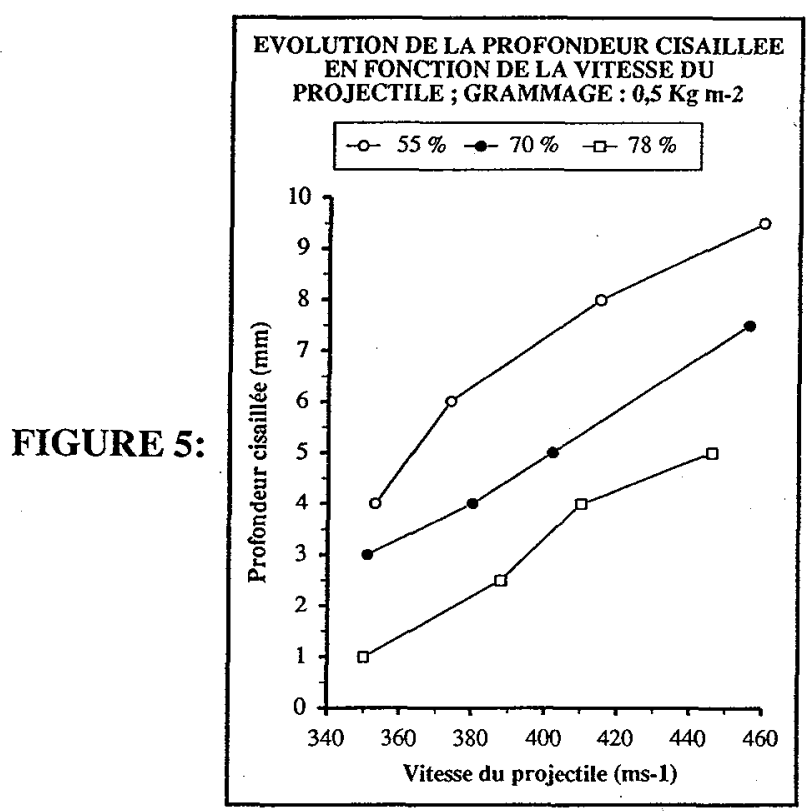

\section{2 - Influence du grammage}

Les tissus fibre de verre sont disponibles à différents grammages. La figure 6 montre qu'une augmentation du nombre de plis par diminution du grammage n'entraîne pas forcément une augmentation de l'énergie absorbée ; îl existe un grammage minimal ici estimé à $0,5 \mathrm{~kg} / \mathrm{m}^{2}$.

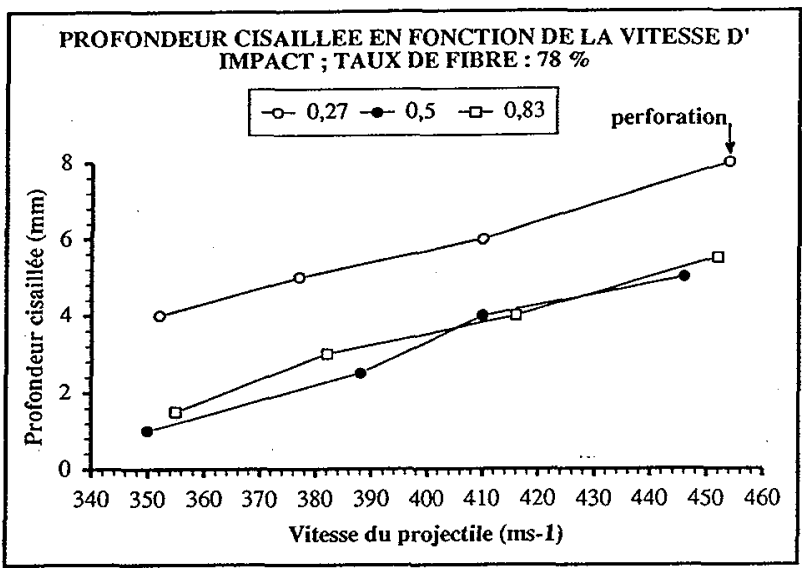

FIGURE 6: 


\section{3 - Relation délaminage-pénétration}

On note sur la figure 7 qu'une augmentation du taux de fibre favorise l'endommagement par délaminage du aux glissements inter-plis.

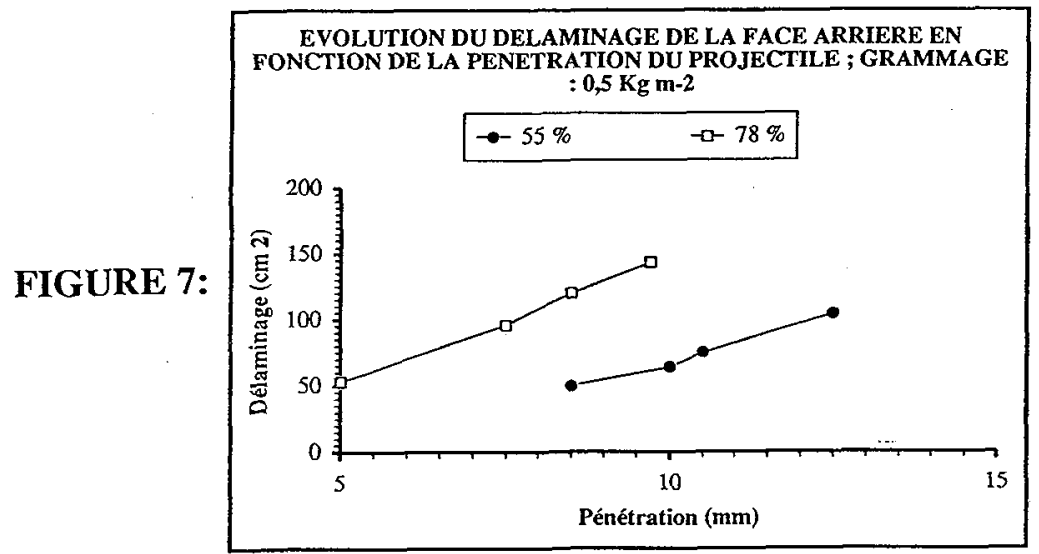

\section{4 - Relation délaminage-déformation}

La déformation permanente de la plaque impactée s'écrit :

$$
\text { déf }=[(n p+p t)-e] / e
$$

La figure 8 montre qu'une grande déformation du matériau est liée à un endommagement important par délaminage.

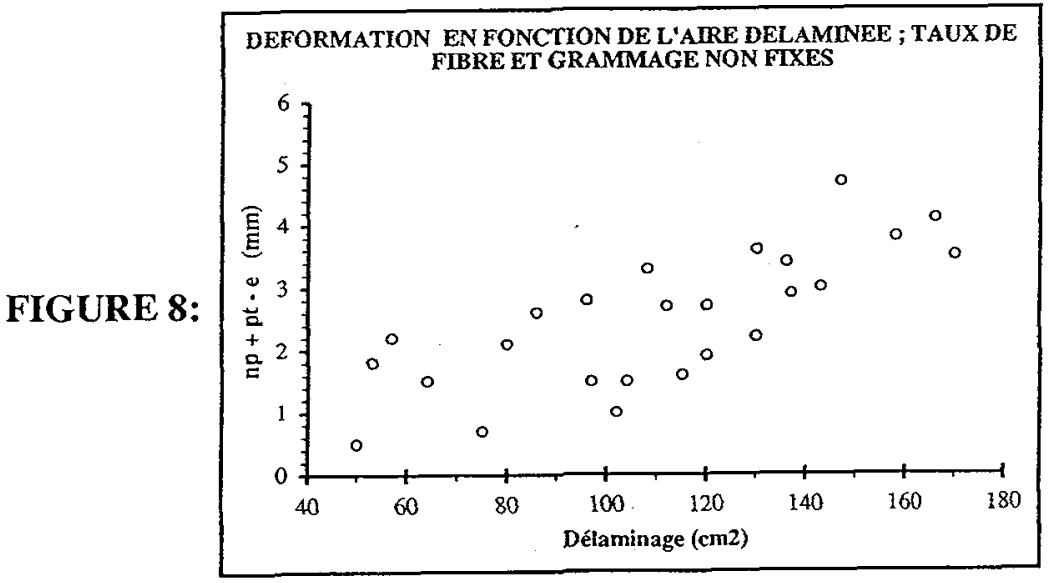

\section{6 - CONCLUSION}

Ces premiers résultats montrent l'intérêt de la méthode d'essai dynamique choisie pour déterminer l'influence des paramètres étudiés.

L'observation microscopique des éprouvettes après essai permet d'identifier les différents mécanismes d'absorption d'énergie mis en jeu.

Il reste à quantifier la contribution de chacun de ces mécanismes à l'énergie totale absorbée au cours de l'impact. 